\title{
A Monte Carlo study for optimizing the detector of SPECT imaging using a XCAT human phantom
}

Mohammad Khoshakhlagh', Jalil Pirayesh Islamian², Seyyed Mohammad Abedi³, Babak Mahmoudian ${ }^{4}$, Masoud Shayesteh Azar ${ }^{5}$ ${ }^{1}$ Immunology Research Center, Tabriz University of Medical Sciences, Tabriz, Iran

2Department of Medical Physics, Faculty of Medicine, Tabriz University of Medical Sciences, Tabriz, Iran

${ }^{3}$ Department of Radiology, Faculty of Medicine, Mazandaran University of Medical Sciences, Sari, Iran

${ }^{4}$ Nuclear Medicine Ward, Department of Radiology-Radiotherapy and Nuclear Medicine, Faculty of Medicine,

Tabriz University of Medical Sciences, Tabriz, Iran

${ }^{5}$ Orthopedic Research Center, Mazandaran University of Medical Science, Mazandaran, Iran

[Received 19 II 2016; Accepted 22 XI 2016]

\begin{abstract}
BACKGROUND: Acquiring a high quality image has assigned an important concern for obtaining accurate diagnosis in nuclear medicine. Detector is a critical component of Single Photon Emission Computed Tomography (SPECT) imaging system for giving accurate information from exact pattern of radionuclide distribution in the target organ. The images are strongly affected by the attenuation, scattering, and response of the detector. The conventional detector is mainly made from sodium iodide activated by thallium $[\mathrm{Nal}(\mathrm{TI})]$ in nuclear medicine imaging.
\end{abstract}

The aim of the study. This study has planned to introduce a suitable for an optimized SPECT imaging. SIMIND Monte Carlo program was utilized for simulating a SPECT imaging system with a Nal(TI) detector, and a low-energy high-resolution (LEHR) collimator.

MATERIAL AND METHODS: The Planar and SPECT scans of a ${ }^{99 m T c}$ point source and also an extended Cardiac-Torso (XCAT) computerized phantom with the experiment and simulated systems were prepared. After verification and validation of the simulated system, the similar scans of the phantoms were compared from the point of view of image quality for 7 scintillator crystals including: $\mathrm{Nal}(\mathrm{TI}), \mathrm{BGO}, \mathrm{YAG}: \mathrm{Ce}, \mathrm{YAP}: \mathrm{Ce}, \mathrm{LuAG}: \mathrm{Ce}, \mathrm{LaBr}_{3}$ and CZT. The parameters of energy and spatial resolution, and sensitivity of the systems were compared. Images were analyzed quantitatively by SSIM algorithm with Zhou Wang and Rouse/Hemami methods, and also qualitatively by two nuclear medicine specialists.

RESULTS: Energy resolutions of the mentioned crystals obtained were: 9.864, 9.8545, 10.229, 10.221, 10.230, 10.131and10.223 percentage for ${ }^{99 \mathrm{mTC}}$ photopeak $140 \mathrm{Kev}$, respectively. Finally, SSIM indexes for the related phantom images were calculated to $0.794,0.738,0.735,0.607,0.760$ and 0.811 compared to the $\mathrm{Nal}(\mathrm{TI})$ acquired images, respectively. Medical diagnosis of the SPECT images of the phantom showed that the system with BGO crystal potentially provides a better detectability for hot and cold lesions in the liver of XCAT phantom.

CONCLUSIONS: The results showed that BGO crystal has a high sensitivity and resolution, and also provides a better lesion detectability from the point of view of image quality on XCAT phantom.

KEY words: gamma detector, SIMIND simulation, SSIM algorithm, XCAT phantom

Nucl Med Rev 2017; 20, 1: 10-14

\section{Background}

Single Photon Emission Computed Tomography (SPECT) has an advantage over Positron Emission Tomography (PET) in that it is less expensive and more widely available, but it is less informa-

Correspondence to: Jalil Pirayesh Islamian

Department of Medical Physics, Faculty of Medicine, Tabriz

University of Medical Sciences, Tabriz, Iran

E-mail: pirayeshj@gmail.com tive in that it has poorer spatial resolution. The images are strongly affected byTab attenuation, scattering, response of the detector and also the detector materials. Conventional detector was mainly made from sodium iodide activated thallium $\mathrm{Nal}(\mathrm{TI})$ in nuclear medicine imaging. Nuclear medicine imaging detector should have some characteristics such as high energy resolution, appropriate photon conversion efficiency, high density, short decay time, short radiation length, as well as the appropriate physical strength, without afterglow, to obtain the radioisotope images with the best qualities for an accurate diagnosis [1]. There are some gamma 
Table 1. Physical properties of some materials of the detectors used in the present study [6, 9-11]

\begin{tabular}{|c|c|c|c|c|c|c|c|}
\hline Properties & Nal:TI & BGO & YAG:ce & YAP:Ce & LuAG:Ce & $\mathrm{LaBr}_{3}$ & CZT \\
\hline Density $\left[\mathrm{g} / \mathrm{m}^{3}\right]$ & 3.67 & 7.13 & 4.55 & 5.37 & 6.76 & 5.78 & 5.08 \\
\hline Index of Refraction & 1.85 & 2.15 & 1.79 & 1.94 & 1.84 & 3.09 & 1.9 \\
\hline Decay Constant (ns) & 230 & 300 & 70 & 25 & 70 & 1 & 26 \\
\hline Light Yield (\%) Nal (TI) & 100 & 25 & 40 & 60 & 20 & - & 160 \\
\hline
\end{tabular}

ray detectors that have been produced and suggested for nuclear medicine imaging [2]. Bismuth Germanate (BGO), may provide a better sensitivity due to its higher density [3], with a low afterglow and is also a non-hygroscopic crystal [1]. BGO crystal was usually used in PET scanners for its high photo fraction that is needed in PET imaging [4]. Moreover, cerium activated Yttrium aluminum garnet (YAG:Ce) and Yttrium aluminum perovskite (YAP:Ce) have the benefits of average density but YAP:Ce has energy resolution better than that of YAG:Ce [5]. On the other hand, YAG:Ce has the ability to detect $\mathrm{X}$-rays and low energy gamma-ray photons. Also, Lutetium aluminum garnet activated by cerium (LuAG:Ce) has higher density than YAG:Ce and faster decay time than BGO crystal [6]. Cerium-activated lanthanum bromide $\left(\mathrm{LaBr}_{3}\right)$ has a good energy resolution and low decay time, high temperature stability also at room temperature [5-7]. Cadmium zinc telluride (CZT), with a high density, very short decay time and an excellent energy resolution recently has been suggested in nuclear medicine imaging system [8]. Therefore, there are some controversy and uncertainty on selecting a preferable detector with the best efficiency in SPECT systems. This study has planned to compare the above mentioned crystals for an optimized SPECT imaging (Table 1) [6, 9-11].

\section{Material and methods}

\section{Monte Carlo simulation}

The Siemens E.CAM gamma camera was modeled using SIMIND Monte Carlo program. The camera consists of a removable low energy high resolution (LEHR) collimator, a Nal (TI) scintillation crystal, a light guide and an array of photomultiplier tubes (PMTs). The parameters of LEHR collimator, used for low energy sources such as ${ }^{99 \mathrm{~m} T c}$, for experiment and simulation were as follows: parallel hexagonal holes with cells of $1.11 \mathrm{~mm}$ diameter, $2.405 \mathrm{~cm}$ height, and $0.16 \mathrm{~mm}$ septal thickness. The Nal (TI) crystal specifications were as follows: planar, $9.5 \mathrm{~mm}$ in thickness, $59.1 \times 44.5 \mathrm{~cm}^{2}$ in area, light yield $40 \mathrm{k}$ photons $/ \mathrm{MeV}$, and a peak emission spectrum at $415 \mathrm{~nm}[12,13]$. Generated light in the crystal is collected by a matrix composed of 59 PMTs, 53 with $7.6 \mathrm{~cm}$ and 6 with $5.1 \mathrm{~cm}$ in diameter. The photocathode is a bialkali type with quantum efficiency of approximately $30 \%$ for the wavelength of maximum $\mathrm{Nal}(\mathrm{TI})$ emission [14].

A light guide ensures a good optical coupling between the scintillating crystal and PMTs. The SIMIND Monte Carlo program was utilized to simulate the aforementioned structures. When ${ }^{99 \mathrm{~m} T \mathrm{C}}$ is used, various structures attached to back of the crystal contribute to backscattering of the emitted photons. To assess the effect of these parts, a single $6 \mathrm{~cm}$ slab of Pyrex was substituted and simulated [15]. SIMIND program mainly consists of two executable programs included: CHANGE.exe, which defines the system and scanning parameters, and SIMIND.exe, which performs the actual simulation [16]. The program can also simulate non-uniform attenuation from voxel-based phantoms and includes several types of variance reduction techniques [16]. The functional parameters of the experiment and simulated systems including energy and special resolution, and energy spectra were compared for verification of the simulated system. Energy resolution of the gamma camera was measured with a ${ }^{99 m} \mathrm{Tc}(3.7 \mathrm{MBq})$ point source, positioned at the center of the field of view (FOV), $25 \mathrm{~cm}$ from the crystal surface. The energy spectrum was acquired for $10^{7}$ photons/projection. Spatial resolution of the real and simulated gamma camera were determined by placing a ${ }^{99 \mathrm{~m} T c}(1.9 \mathrm{MBq})$ source $(1.5 \mathrm{~mm}$ in diameter) at the center of the FOV. A study of the SPECT reconstructed spatial resolution was also carried out both experimentally and by SIMIND simulation. SPECT projections of a Jaszczak Deluxe Phantom along the axis of rotation were acquired [17].

The program was also used for simulation of the SPECT system with CZT, LaBr3, LuAG:Ce, YAG:Ce, YAP:Ce and BGO crystals and related phantom studies.

\section{Phantom studies}

A point source of $3.7 \mathrm{MBq}^{99 \mathrm{~m}} \mathrm{Tc}, \mathrm{Na}^{99 \mathrm{~m}} \mathrm{TcO}_{4}$ solution, and a Jaszczak phantom uniformly filled with $370 \mathrm{MBq}^{99 \mathrm{~m} T c}$, respectively at 10 and $15 \mathrm{~cm}$ from a LEHR collimator, were used both for the experiment and simulation studies, a detailed description was delivered in the previous study by Khoshakhlagh et al. [24]. The produced images were compared in terms of image contrast and spatial resolution (Figure 1) [19-21]. Moreover, for the XCAT phantom studies, the acquisition parameters were $128 \times 128$ matrix, 128 views, $3.12 \mathrm{~mm}$ pixel size, zoom 1.2, and the projections were reconstructed by filtered back projection reconstruction method using a Butterworth filter with a cut-off frequency of 0.5 .

\section{Image evaluation}

\section{Quantitative study}

The structural similarity (SSIM) algorithm was used for quantitative study on image quality $[22,23]$. The algorithm has been described in details in previous study by Islamian et al. [20].

\section{Qualitative study}

For a qualitative study on image quality, two nuclear medicine specialists viewed the filtered back projection reconstructed radioisotope images for the lesion detectability of a hot and a cold lesion, both in sizes of $10 \mathrm{~mm}$ diameter, in the liver of XCAT human phantom and scaled the images quality from 1 to 9 so that 


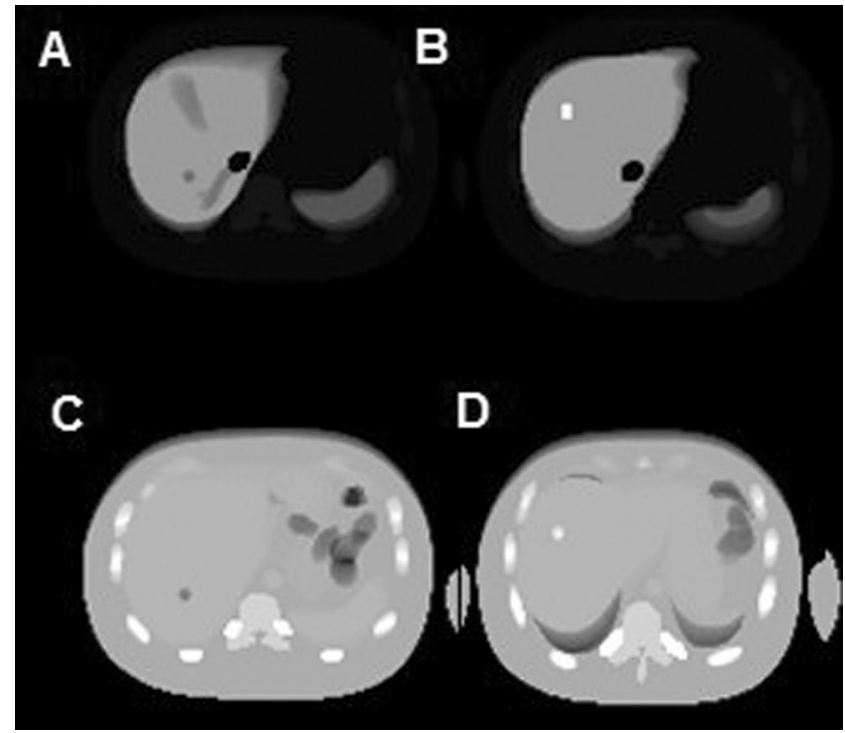

Figure 1. The cross sections of the simulated Extended Cardiac-Torso (XCAT) computerized human phantom. Liver activity maps with a cold lesion (A), and a hot lesion (B). The related attenuation maps with a low attenuation lesion (C) and a high attenuation lesion (D)

Table 2. The results of simulation verification according to the functional parameters of the experiment and simulated SPECT systems for a ${ }^{99 m} \mathrm{Tc}$ point source scanning

\begin{tabular}{lcc}
\hline Parameter & Experiment system & Simulated system \\
\hline Spatial Resolution [mm] & 7.524 & 7.287 \\
Energy Resolution (\%) & 9.864 & 9.192
\end{tabular}

number 9 was marked for the best detectability and the worst one was acquired number 1 . The letters $\mathrm{H}$ and $\mathrm{C}$ were used for the hot lesion and the cold lesion detectability, respectively.

\section{Results}

\section{Point source}

The verification of the simulated SPECT system according to the functional parameters of the systems was done by a ${ }^{99 m} \mathrm{Tc}$ point source scanning, according to the previous study by Khoshakhlagh et al. [24] (Table 2).
Some minor differences may be observed between the simulated and the experimental energy spectra, the most striking being that the experimental spectrum presents a wider peak which may be explained by the superimposition of the energy peaks with the X-ray energy of ${ }^{99 m} \mathrm{Tc}$ [25].

\section{XCAT phantom}

The data on comparing the images of SPECT scanning of XCAT phantom with $\mathrm{Nal}(\mathrm{TI}), \mathrm{CZT}$, LaBr3, LuAG:Ce, YAG:Ce, YAP:Ce and BGO crystals by SSIM algorithm with Zhou Wang and Rouse/Hemami methods are presented in Table 3. Figure 2 shows the detectability of liver lesion in the simulated XCAT phantom acquisitions by SPECT imaging with the seven detectors and also Figure 3 shows the related results on the interpretations of the filtered back projection images by two nuclear medicine specialists.

\section{Discussion}

The present study was planned to possibly replace the conventional scintillation detector, $\mathrm{Nal}(\mathrm{TI})$, with the newly provided detectors for more efficient detection and higher resolution. We simulated a SPECT imaging system with a Nal(TI) detector crystal, also replaced with six crystals including BGO, YAG:Ce, YAP:Ce, LuAG:Ce, $\mathrm{LaBr}_{3}$ and CZT, and compared the related images quality in SPECT scans of XCAT human phantom simulated with hot and cold lesions in the liver. Our study demonstrated that a SPECT system equipped with a BGO detector is somewhat more efficient for detecting the lesions compared to the others. In a previous in-vivo

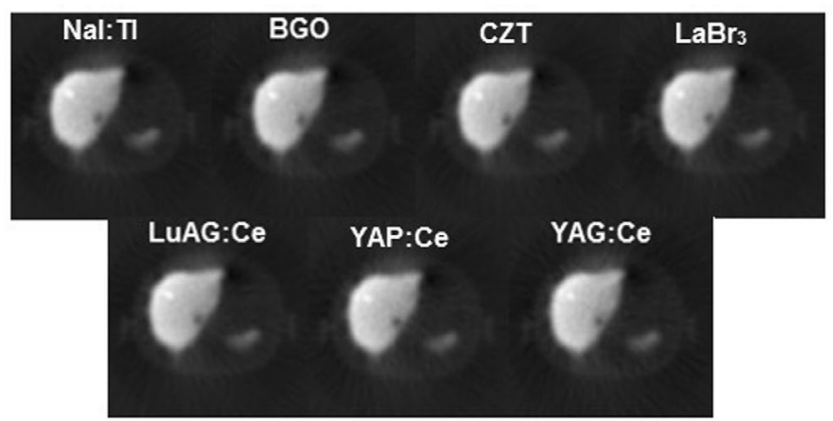

Figure 2. Radioisotope images of the simulated SPECT from XCAT human phantom acquisition (liver as the target organ), by the seven detectors. The scan parameters were described in the text

Table 3. The data on comparing the acquired images from SPECT scans of XCAT human phantom with six detectors by SSIM algorithm, compared to the $\mathrm{Nal}(\mathrm{Tl})$

\begin{tabular}{|c|c|c|c|c|c|c|c|}
\hline \multirow[t]{2}{*}{ Method } & \multirow[t]{2}{*}{ Parameter } & \multicolumn{6}{|c|}{ Detector } \\
\hline & & BGO & YAG:ce & YAP:Ce & LuAG:Ce & $\mathrm{LaBr}_{3}$ & CZT \\
\hline \multirow[t]{4}{*}{ Zhou Wang } & Luminance comparison & 1.000 & 0.987 & 0.997 & 0.981 & 0.999 & 1.000 \\
\hline & Contrast comparison & 0.977 & 0.965 & 0.965 & 0.920 & 0.973 & 0.977 \\
\hline & Structure comparison & 0.812 & 0.77485 & 0.763 & 0.672 & 0.781 & 0.830 \\
\hline & MS-SSIM index Zhou Wang & 0.794 & 0.73854 & 0.735 & 0.607 & 0.759 & 0.811 \\
\hline \multirow{3}{*}{ Rose Hemmami } & Contrast comparison & 0.954 & 0.93953 & 0.937 & 0.946 & 0.945 & 0.956 \\
\hline & Structure comparison & 0.683 & 0.64980 & 0.623 & 0.673 & 0.645 & 0.721 \\
\hline & MS-SSIM index Rouse/Hemami & 0.651 & 0.602 & 0.582 & 0.637 & 0.609 & 0.689 \\
\hline
\end{tabular}




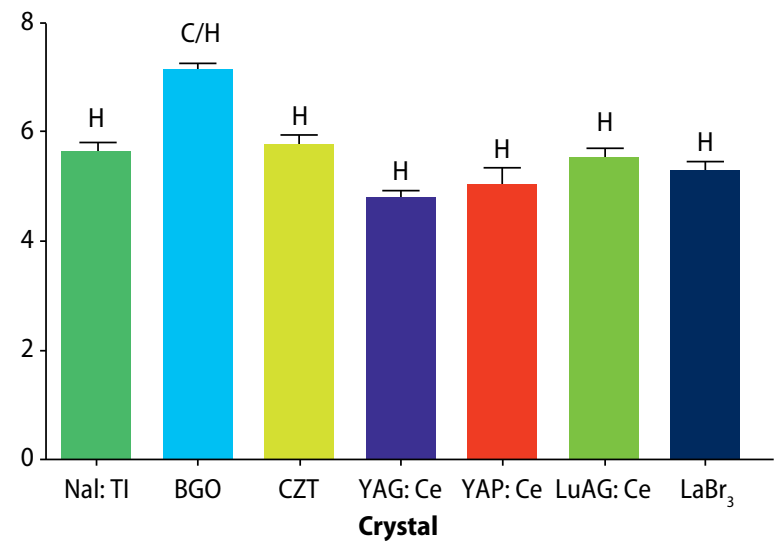

Figure 3. The results on detectability of the hot $(\mathrm{H})$ and cold $(\mathrm{C})$ lesions in reconstructed radioisotope SPECT images of XCAT liver by the seven detectors

study by Saizu et al., on the image quality acquired from a LaBr3 crystal for administration of $\mathrm{I}-131$ in the thyroid the $\mathrm{LaBr}_{3}$ has been proposed as an appropriate detector [7]. Herein we also obtained some better resolution for that detector compared to the $\mathrm{Nal}(\mathrm{TI})$, but not better than for BGO. The properties that make the $\mathrm{LaBr}_{3}$ :Ce scintillator detector attractive for different applications, based on $\gamma$-ray spectrometry, include its very suitable energy resolution. Furthermore, Derenzo et al. [26], have also compared the $\mathrm{Nal}(\mathrm{TI})$ and $\mathrm{BGO}$ detectors and found that the latter is more sensitive mainly due to its higher density. Meanwhile, in a review by Liu B et al., on the development of medical scintillators, it was demonstrated that BGO crystal has occupied far more than 50\% PET market [1]. Our results also confirmed the mentioned studies and showed that, between the studied BGO, YAG:Ce, YAP:Ce, LuAG:Ce, $\mathrm{LaBr}_{3}$ and CZT crystals, BGO provides a higher sensitivity with some suitable energy resolution. Also, comparing the results of the images quality from the point of lesion detectability view, our study showed that the images acquired by the BGO crystal provide some better qualities in detecting hot and cold lesions in the liver of XCAT human phantom (Figure 3). Finally, BGO benefits from low afterglow and so it should also be preferred in high count rate applications. These causes reduced pulse pile up and increased energy resolution in radionuclide imaging.

\section{Conclusion}

Lesion detectability is a challenging study in SPECT imaging, and the detector plays a critical role in providing a high resolution projections. This SIMIND Monte Carlo simulation study on the determination of an optimized crystal for SPECT imaging proposed that the BGO may candidate as a suitable detector for detecting of hot and cold lesions in the liver of XCAT human phantom. However, the present study should be more extended by including semiconductors and also the influence of parameters such as thickness of the detector on obtaining higher efficency and optimal imaging quality.

\section{Acknowledgements}

The work has been funded by the Immunology Research Center of Tabriz University of Medical Sciences, Tabriz, Iran (Grant \# 5.47.2594). The authors have special thanks to Professor Michael
Ljungberg for providing the files of the detector materials and the related interaction cross sections for the SIMIND simulation and also to the department of Nuclear Medicine in Fatemeh Al-Zahra Hospital of Sari, Iran.

\section{References}

1. Liu B, Shi C. Development of medical scintillator. J Chin Sci Bull. 2002; 47(13): 1057-1063.

2. Melcher $\mathrm{CL}$. Perspectives on the future development of new scintillators. Nucl Instrum Methods Phys Res A. 2005; 537(1-2): 6-14, doi: 10.1016/j. nima.2004.07.222.

3. Crystals.saint-gobain.com [homepage on the Internet]. BGO Bismuth germanates scintillation material. http://www.crystals.saint-gobain. com/uploadedFiles/SG-Crystals/Documents/BGO\%20data\%20sheet.pdf (Last accessed 2016 Oct 13)

4. Rahmim A, Zaidi H. PET versus SPECT: strengths, limitations and challenges. Nucl Med Commun. 2008; 29(3): 193-207, doi: 10.1097/MNM.0b013e3282f3a515, indexed in Pubmed: 18349789.

5. Moszynski M, Kapusta M, Zalipska J, et al. Low energy -rays scintillation detection with large area avalanche photodiodes. IEEE Trans Nucl Sci. 1999; 46(4): 880-885, doi: 10.1109/23.790697.

6. Chewpraditkul W, Swiderski L, Moszynski M, et al. Scintillation Properties of LUAG:Ce, YAG:Ce and LYSO:Ce Crystals for Gamma-Ray Detection. IEEE Transactions on Nuclear Science. 2009; 56(6): 3800-3805, doi: 10.1109/tns.2009.2033994.

7. Saizu MA, Cata-Danil G. Lanthanum Bromide Scintillation Detector for Gamma Spectrometry Applied in Internal Radioactive Contamination Measurements. UPB Sci Bull, Series A. 2011; 73(3): 119-126.

8. Gehealthcare.com [homepage on the Internet]. CZT Technology: Fundamentals and Applications. http://www3.gehealthcare.com/en/products/categories/nuclear_medicine/cardiac_imaging/discovery_nm570c. (Last accessed 2016 Oct 13).

9. Levin CS. New Imaging Technologies to Enhance the Molecular Sensitivity of Positron Emission Tomography. Proceedings of the IEEE. 2008; 96(3): 439-467, doi: 10.1109/jproc.2007.913504.

10. Eijk C. Inorganic scintillators in medical imaging. Phys Med Biol. 2002; 47(8): R85-R106, doi: 10.1088/0031-9155/47/8/201.

11. Dorenbos P. Light output and energy resolution of Ce3+-doped scintillators. Nucl Instrum Methods Phys Res A. 2002; 486(1-2): 208-213, doi: 10.1016/s0168-9002(02)00704-0.

12. Motta D, Schönert S. Optical properties of bialkali photocathodes. Nucl Instrum Methods Phys Res A. 2005; 539(1-2): 217-235, doi: 10.1016/j. nima.2004.10.009.

13. Data sheet of Siemens E.CAM Dual Head gamma camera. 1992. p.1-12.

14. Vittori F, Notaristefani Fde, Malatesta T. Crystals and light collection in nuclear medicine. Nuclear Physics B - Proceedings Supplements. 1999; 78(1-3): 616-621, doi: 10.1016/s0920-5632(99)00614-3.

15. De Vries DJ, Moore SC, Zimmerman RE, et al. Development and validation of a Monte Carlo simulation of photon transport in an Anger camera. IEEE Trans Med Imaging. 1990; 9(4): 430-438, doi: 10.1109/42.61758, indexed in Pubmed: 18222790.

16. SIMIND Monte Carlo program. http://www.msf.lu.se/forskning/the-simind-monte-carlo-program (Last accessed 2016).

17. Groch MW, Erwin WD. Single-photon emission computed tomography in the year 2001: instrumentation and quality control. J Nucl Med Technol. 2001; 29(1): 12-18, doi: 10.1007/springerreference_124034, indexed in Pubmed: 11283212.

18. Islamian JP, Toossi MT, Momennezhad M, et al. Simulation of a Quality Control Jaszczak Phantom with SIMIND Monte Carlo and Adding the Phantom as an Accessory to the Program. Iran J Med Phys. 2012; 9(2): 135-140, doi: 10.22038/ijmp.2012.319. 
19. Segars WP, Sturgeon G, Mendonca S, et al. 4D XCAT phantom for multimodality imaging research. Med Phys. 2010; 37(9): 4902-4915, doi: 10.1118/1.3480985, indexed in Pubmed: 20964209.

20. Islamian JP, Toossi MT, Momennezhad M, et al. Monte Carlo study of the effect of collimator thickness on T-99m source response in single photon emission computed tomography. World J Nucl Med. 2012; 11(2): 70-74, doi: 10.4103/1450-1147.103419, indexed in Pubmed: 23372440.

21. Islamian JP, Toossi MB, Momennezhad M, et al. Monte Carlo Study of the Effect of Backscatter Material Thickness on 99mTc Source Response in Single Photon Emission Computed Tomography. Iran J Med Phys. 2013; 10(1): 69-77, doi: 10.22038/ijmp.2013.920.

22. Rouse D, Hemami S. Analyzing the role of visual structure in the recognition of natural image content with multi-scale SSIM. Human Vision and Electronic Imaging XIII. 2008, doi: 10.1117/12.768060.
23. Wang Z, Bovik AC, Sheikh HR, et al. Image quality assessment: from error visibility to structural similarity. IEEE Trans Image Process. 2004; 13(4): 600-612, doi: 10.1109/tip.2003.819861, indexed in Pubmed: 15376593.

24. Khoshakhlagh M, Islamian JP, Abedi M, et al. A Study on Determination of an Optimized Detector for Single Photon Emission Computed Tomography. World J Nucl Med. 2016; 15(1): 12-17, doi: 10.4103/1450-1147.167588, indexed in Pubmed: 26912973.

25. Toossi MB, Islamian PJ, Momennezhad M, Ljungberg M, Naseri S. SIMIND Monte Carlo simulation of a single photon emission CT. J Med Phys 2010; 35(1): 42-7.

26. Derenzo S, Budinger T, Huesman R, et al. Imaging Properties of a Positron Tomograph with 280 Bgo Crystals. IEEE Trans Nucl Sci. 1981; 28(1): 81-89, doi: 10.1109/tns. 1981.4331144 